\title{
Solar Fluctuations and the MSW Effect
}

\author{
C.P. Burgess \\ Physics Department, McGill University, 3600 University Street, \\ Montréal, Québec, Canada, H3A $2 T 8$.
}

\begin{abstract}
This talk summarizes the results of recent calculations of how fluctuations within the solar medium can influence resonant neutrino oscillations within the sun. Although initial calculations pointed to helioseismic waves as possibly producing detectable effects, recent more careful calculations show this not to be true. Those features of fluctuations which maximize their influence on neutrino propagation are identified, and are likely to have implications for supernovae and the early universe.
\end{abstract}

\section{Introduction}

Nhe results from solar-neutrino experiments [1] now strongly suggest that the experimentally-observed solar-neutrino spectrum differs from the predictions of astrophysical theory [2]. The observed spectrum Is consistent with a virtually complete elimination of the flux of intermediate-energy ${ }^{7}$ Be neutrinos,

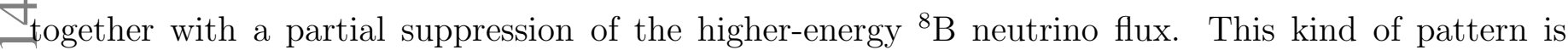
notoriously difficult to obtain using astrophysical variations on the solar-model theme (notwithstanding the recent proposal of significant ${ }^{3} \mathrm{He}$ convection [3]), suggesting instead an interpretation based on nonstandard neutrino properties. Perhaps the most successful such neutrino-based explanation is the QMSW mechanism [田], which proposes the resonant conversion of neutrino flavours within the solar miedium, providing an excellent description of the experiments using theoretically-plausible neutrino parameters.

The emergence of the MSW mechanism as the leading explanation for the solar-neutrino problem has motivated more detailed scrutiny of the approximations on which its predictions rest. For example, the original MSW analysis starts from a mean-field treatment of the background through which the neutrinos propagate. More recent work has since investigated how corrections to the mean-field picture might influence the neutrino survival probability [5, 6, 7, 8, 9, 10]. These studies indicate that there are astrophysical situations for which fluctuations might significantly influence neutrino propagation despite the extreme weakness of neutrino interactions. The two different ways in which they might do so are:

1. Fluctuations can scatter neutrinos away from the forward direction, and so shorten the effective neutrino mean free path a the medium.

2. For situations where the mean particle densities cause resonant oscillations amongst neutrino flavours, fluctuations generically act to ruin the quality of the resonance. 
Explorations of the practical implications of these effects are in their infancy. Naturally enough, the first applications have been to solar neutrinos, for which the most experimental information is available. They have therefore focussed on the second of the alternatives listed above, since the density of the solar medium precludes seeing the consequences of direct neutrino scattering from fluctuations.

By contrast, MSW oscillations, if they occur, hold out the most promising way of seeing the effects of solar fluctuations on neutrino propagation. This is because even though the fluctuation effects are small, resonant oscillations make neutrinos more sensitive to fluctuations than they would otherwise be. Most interestingly, independent preliminary calculations $\llbracket 7$, 8 indicated that reasonblysized fluctuations in the solar interior might be detectable through the deterioration of the MSW resonance which they induce.

The next sections contain a summary of these estimates as well as subsequent, more precise, calculations [10] of the effects of known solar fluctuations on neutrino propagation. In so doing we follow the presentation of refs. [8] and [10], with which we are most familiar. $§ 2$ summarizes which features fluctuations must have in order to appreciably affect neutrino oscillations. For solar applications, helioseismic waves emerge as the fluctuations with the best chance of spoiling the MSW effect. §3 then briefly describes the results of detailed simulations of neutrino oscillations in the presence of helioseismic waves, culminating with the conclusion that no known source of solar fluctuations is large enough to have a detectable influence on solar neutrinos.

\section{General Features}

Much is known about how particles propagate through materials, and general techniques have been developed to describe this propagation. This section describes the adaptation of these ideas to neutrino physics, as developed in ref. [8].

\subsection{The Origin of Fluctuations}

Since neutrinos interact so weakly with the solar medium, they are negligibly scattered by microscopic effects such as thermal fluctuations. As a result, the time evolution of the density matrix, $\rho$, describing the neutrino state is given by the usual quantum-mechanical expression:

$$
\rho(t, n)=U(t, n) \rho(0) U^{\dagger}(t, n)
$$

where $t$ denotes time, $U(t, n)$ is the evolution operator, and $n$ generically denotes all of those features of the solar medium (like the local electron density profile) on which neutrino evolution can depend

\footnotetext{
${ }^{1}$ See, however, ref. [11] for a discussion of some fluctuation effects within supernovae.
} 
parametrically. For neutrino oscillation experiments we take $\rho$ to be a matrix in neutrino-flavour space, and $U$ is the solution to the evolution equation

$$
\frac{\partial U}{\partial t}=-i V(t, n) U
$$

where $V(t, n)$ is the interaction Hamiltonian which couples the neutrinos to each other and to the solar medium. For example, for an ultrarelativistic neutrino having three-momentum $k$ and mass matrix $m$ propagating through the sun, the dominant flavour-dependent interactions are $V=V_{\text {vac }}+V_{\text {mat }}(t, n)$, with:

$$
\begin{aligned}
V_{\mathrm{vac}} & \approx \frac{m^{\dagger} m}{2 k}+\ldots \\
V_{\mathrm{mat}}(t, n) & \equiv \sqrt{2} G_{F} g^{e} n_{e}(t)
\end{aligned}
$$

where $g^{e}=\operatorname{diag}(1,0)$ is a matrix describing the charged-current coupling to the electron density $n_{e}$. For simplicity we consider here only the case of two active neutrino species.

For the present purposes fluctuations arise when the electron density, $n_{e}$, is not constant in space or time, since then the solar properties as seen along the trajectories of successive neutrinos can vary. The response of a detector to many such neutrinos is then described by an average over the ensemble of densities which are seen by the individual particles, weighted by some probability distribution, $p(n)$ :

$$
\langle\mathcal{O}\rangle(t)=\int d n p(n) \operatorname{Tr}[\mathcal{O} \rho(t, n)]=\operatorname{Tr}[\mathcal{O}\langle\rho(t)\rangle]
$$

The physics of the fluctuations is encoded in the probability distribution, $p(n)$, which we define in terms of the expansion of $n_{e}$ in a complete set of basis functions, $\phi(t)$, that are assumed to describe uncorrelated variables. That is:

$$
n_{e}(t)=\left\langle n_{e}(t)\right\rangle\left[1+\sum_{j} \mathcal{C}_{j} \phi_{j}(t)\right],
$$

where the coefficients $\mathcal{C}_{j}$, are assumed to be uncorrelated random variables which are Gaussian distributed, with vanishing mean: $\left\langle\mathcal{C}_{j}\right\rangle=0$ and $\left\langle\mathcal{C}_{j} \mathcal{C}_{k}\right\rangle=\sigma_{j}^{2} \delta_{j k}$

The most commonly-used choice of basis functions [6, 7, 8] are localized in space, corresponding to fluctuations which are uncorrelated from point to point. Since this choice is not appropriate for helioseismic waves, for these we instead follow refs. [8, 10] and expand the electron density in terms of a complete basis of helioseismic modes [12].

The brute force approach is to directly solve these equations for $U(t, n)$ and $\rho(t, n)$, to find the electron-neutrino survival probability for any one neutrino after its transit through the sun, and to then numerically average the result over the appropriate ensemble. Before describing the results so obtained, we first pause to describe an analytic solution which applies in the limit that the correlation lengths describing the fluctuations are much smaller than all other scales in the problem. 


\subsection{Short Correlation Lengths: The Master Equation}

It pays to recognize that the neutrino-oscillation problem in a random medium necessarily involves (at least) two important time scales. This pays because the time-evolution greatly simplifies if one of these scales should be much smaller than the other. All of the calculations of solar fluctuations, apart from ref. [10], are based on this simplifying assumption. This section describes how this approximation works.

One of the basic scales for neutrino oscillations is the oscillation time, $\tau_{\text {osc }}$, which we define to be the time required for different neutrino flavours to acquire significantly different phases as they evolve through the medium. By definition, oscillation phenomena always involve evolution over scales $t>\tau_{\text {osc }}$, which cannot be computed perturbatively in the interactions which distinguish between the oscillating neutrino species.

The randomness of the interaction between the neutrino and its environment, described above, introduces the other important timescale. More precisely, denoting $\delta V=V-\langle V\rangle$, suppose that the correlation $\left\langle\delta V(t) \delta V\left(t^{\prime}\right)\right\rangle$ is negligible whenever $\left|t-t^{\prime}\right|$ is greater than some characteristic scale, $\tau_{c}$. In this case the neutrino density matrix at time $t, \rho(t)$, retains a memory of the fluctuations through which it has passed only over the time interval $\left(t-\tau_{c}, t\right)$.

The simplification occurs if $\tau_{c} \ll \tau_{\text {osc }}$. In this case, the evolution from an initial state, $\rho(t)$, to a state at a later time, $\rho(t+\Delta t)$, can be computed in perturbation theory:

$$
\Delta \rho \equiv \rho(t+\Delta t)-\rho(t) \equiv \frac{D \rho}{D t} \Delta t
$$

so long as $\Delta t \ll \tau_{\text {osc }}$. Here $D \rho / D t$ defines a coarse-grained time derivative, which may be computed as an explicit convolution of $\rho$ and $V$ over the time interval $(t, t+\Delta t)$.

On the other hand, if $\Delta t$ can also be chosen to satisfy $\Delta t \gg \tau_{c}$, then $D \rho / D t$, evaluated at time $t$, depends only on the initial value of $\rho(t)$, and has no memory of how $\rho$ has evolved over earlier times. This removes the complication of having $D \rho / D t$ involve a convolution over the entire time interval, $(t, t+\Delta t)$. For example, as applied to the neutrino density matrix in two-by-two flavour-space, the twin conditions $\tau_{c} \ll \Delta t \ll \tau_{\text {osc }}$ lead to:

$$
\frac{D \rho}{D t}=-i\left[V_{\text {vac }}+V_{M S W}(t), \rho\right]-2 G_{F}^{2} \mathcal{A}(t)\left[\left(g^{e}\right)^{2} \rho+\rho\left(g^{e}\right)^{2}-2 g^{e} \rho g^{e}\right]+\mathcal{O}\left(V^{3}\right)
$$

where $V_{M S W}(t, n)=\sqrt{2} G_{F} g^{e}\left\langle n_{e}(t)\right\rangle$ and the coefficient $\mathcal{A}(t)$ of the second term is the correlation integral

$$
\mathcal{A}(t) \equiv \int_{t^{\prime}}^{t} d \tau\left\langle\delta n_{e}(t) \delta n_{e}(\tau)\right\rangle
$$

which represents fluctuation effects. Notice that the first term on the right-hand-side of eq. (7) describes the usual MSW evolution of neutrino flavour. 
Now comes the main point. Eqs. (6) and (7) — the Master Equations — define a Markov-like process which gives $\partial \rho(t) / \partial t \approx D \rho / D t$ purely in terms of $\rho(t)$ and $V(t)$. This relation may be directly integrated to obtain $\rho\left(t^{\prime}\right)$ for any later $t^{\prime}$. The result holds even for $\left|t^{\prime}-t\right| \gg \tau_{\text {osc }}$, even though $D \rho / D t$ is only computed perturbatively in $V$.

For two-flavour neutrino oscillations this master equation, including fluctuations, may be solved analytically subject to the same assumptions as are typically used to solve the MSW oscillation problem [8]. The resulting expression for the electron-neutrino survival probability is a generalization of the well-known Parke formula [13] to include the effects of fluctuations:

$$
P_{e}\left(t, t^{\prime}\right)=\frac{1}{2}+\left(\frac{1}{2}-P_{J}\right) \lambda \cos 2 \theta_{m}\left(t^{\prime}\right) \cos 2 \theta_{m}(t)
$$

Here $P_{J}=\exp \left[-\frac{\pi}{2}\left(\frac{\sin ^{2} 2 \theta_{V}}{\cos 2 \theta_{V}}\right)\left(\frac{\delta m^{2} h}{2 k}\right)\right]$ is the 'jump' probability, where $h$ is the scale height for $\left\langle n_{e}(t)\right\rangle$, and $\delta m^{2}$ is the squared-mass difference between the two neutrino mass eigenstates in vacuum. $\theta_{V}$ is the vacuum mixing angle, while $\theta_{m}(t)$ is the matter mixing angle evaluated at the position occupied by the neutrino at time $t$. The contributions of fluctuations are summarized by the coefficient $\lambda$, which is given by

$$
\lambda \equiv \exp \left[-2 G_{F}^{2} \int_{t^{\prime}}^{t} d \tau \mathcal{A}(\tau) \sin ^{2} 2 \theta_{m}(\tau)\right]
$$

An important consequence of this equation is its implication that the probability of $\nu_{e}$ survival depends almost exclusively on fluctuation properties at the position of the MSW resonance, $\operatorname{since} \sin ^{2} 2 \theta_{m}$ is typically sharply peaked at this point.

Figure 1 plots the survival probability vs neutrino energy which is predicted for two particular models of solar density fluctuations. Successive curves in this plot represent fluctuations having different values for their correlation length, $\ell$, and their relative amplitude, $\epsilon^{2} \sim\left\langle\delta n_{e} \delta n_{e}\right\rangle /\left\langle n_{e}\right\rangle^{2}$ at the position where the MSW resonance occurs.

\section{Figure 1}

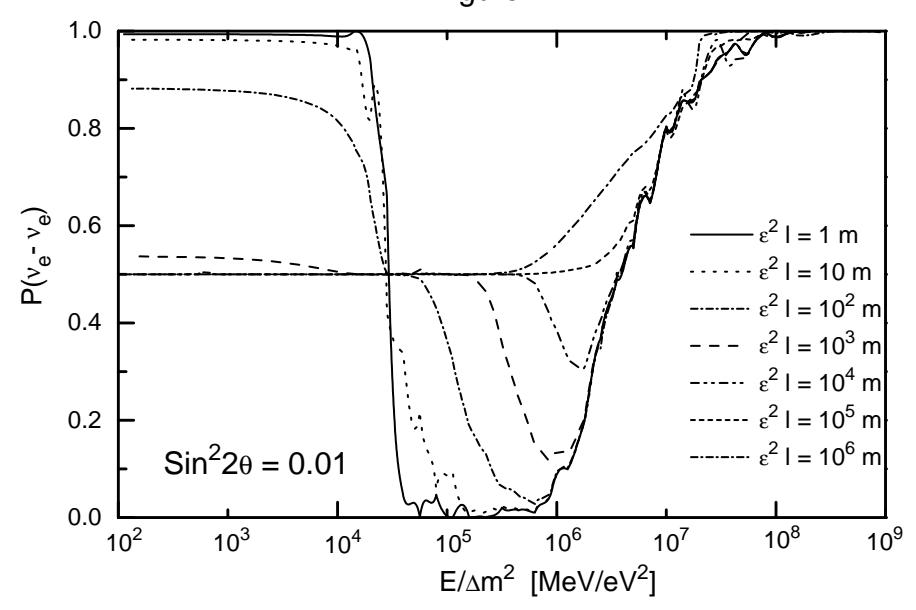

Figure 1: The electron-neutrino survival rate vs neutrino energy as predicted by the Master-equation method using a particular model of solar fluctuations, and representative solar-neutrino properties. Each curve represents a fluctuation having a different value for the one relevant combination of amplitude, $\epsilon$, and correlation length, $\ell$. See ref. 8 for details. 


\subsection{Long Correlation Lengths}

The generalized Parke formula provides an excellent description of neutrino oscillations in media having short-correlation-length fluctuations, as has been verified by comparing it with direct numerical integrations [8]. Two important questions remain, however, which the present section addresses. First, how badly does it fail when $\tau_{c}$ is not small enough to justify the Master-equation approach? Second, for realistic solar neutrinos what does the condition $\tau_{c} \ll \tau_{\text {osc }}$ translate into for the correlation length of a candidate fluctuation in the sun?

Anwering the second of these first, for solar neutrinos the correlation time, $\tau_{c}$, represents the time taken for a neutrino to traverse the correlation length, $\ell$, of a solar fluctuation. The numerical comparisons and analytical estimates of ref. [10] indicate that the Master equation may be expected to work so long as $\ell$ satisfies $\ell \lesssim \ell_{\text {crit }}=1 /\left(\epsilon G_{F}\left\langle n_{e}\right\rangle\right)$ (recall $\epsilon^{2} \sim\left\langle\delta n_{e} \delta n_{e}\right\rangle /\left\langle n_{e}\right\rangle^{2}$ is a measure of the relative amplitude of the fluctuation at the position where the MSW resonance occurs). Taking $\epsilon \sim 10 \%$ for the fluctuation amplitude and $\ell_{\text {crit }} \sim 300 \mathrm{~km}$, corresponding to the electron density at the resonance point, this condition becomes $\ell \lesssim 3,000 \mathrm{~km}$.

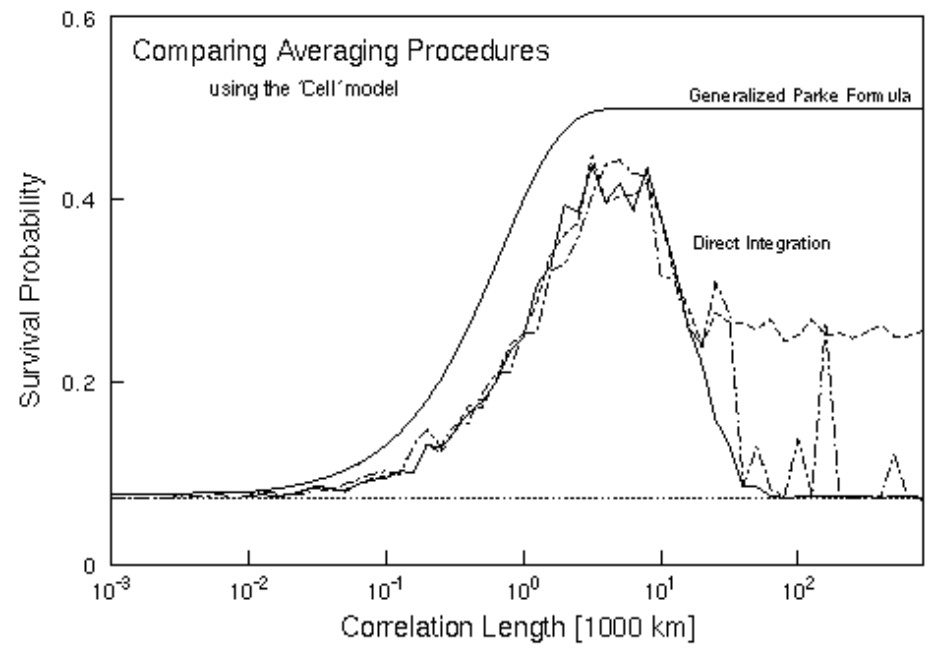

Figure 2: A comparison of the generalized Parke formula with the results of several numerical averages over a particular model - the 'Cell' model - of solar fluctuations. The survival probability is plotted as a function of the correlation length, $\ell$, using the representative values for solar neutrinos. See ref. [10] for details.

How badly does the Master equation describe fluctuations which are larger than this? Very badly indeed. Figure 2 shows a comparison of the generalized Parke formula with a series of direct numerical evaluations of neutrino evolution and the ensemble average, as reported in ref. [10]. As may be seen from the figure, fluctuation effects completely disappear once $\ell$ passes appreciably above $\ell_{\text {crit }}$.

There is a qualitative argument for why fluctuations with very long correlation lengths should not influence resonant neutrino conversion. A density fluctuation in the extreme limit $\ell \sim R_{\odot}$, simply 
corresponds to an overall shift of the electron density throughout the entire sun. This has the sole effect of simply moving the position within the sun where the the resonance occurs, but does not alter the overall survival probability.

In summary, the lessons to be drawn from the extant calculations of neutrino oscillations within a fluctuating medium are these:

1. Master equation methods describe solar neutrinos well for sufficiently small correlation lengths, but can dramatically overestimate the deviation from the MSW prediction when correlation lengths are large. For solar neutrinos, the dividing line between large and small correlation lengths is of order $3,000 \mathrm{~km}$.

2. Fluctuations can cause an appreciable deterioration of the MSW resonance only if two conditions are satisfied. First, fluctuation amplitudes cannot be too small. Second, their correlation lengths should be neither too big nor too small compared to the neutrino oscillation length. Most importantly, both of these conditions must apply at the positions in the sun where the neutrino passes through the MSW resonance.

3. There is only one class of fluctuations in the sun which are known to exist with interestingly large amplitudes deep within the solar radiative zone, where the MSW resonance occurs. These are helioseismic waves [12], for which the correlation lengths at resonance can be as large as $R_{\odot} / 10 \sim 70,000 \mathrm{~km}$. As such they easily exceed the size of $\ell_{\text {crit }}$, and so preclude a description using only Master-equation techniques.

\section{Helioseismic Waves}

We now summarize the results of detailed simulations of neutrino oscillations in the presence of helioseismic waves, as was originally reported in ref. [10]. This reference explicitly computes seismic wave profiles within the sun in order to reliably correlate their amplitude at the resonance point with observations (which are made at the solar surface). It then numerically evolves neutrinos through these profiles to identify their effect on MSW oscillations. No use is made of the short-correlation-length approximation.

There is a simple picture of what is going on. For any particular neutrino, microscopic fluctuations are irrelevant, and the perceived density profile is static since the time taken for a neutrino to traverse the sun (several seconds) is much shorter than the typical period of a helioseismic wave (minutes to hours). The oscillations of each neutrino are therefore well described by the ordinary Parke formula, i.e. eq. (9) with $\lambda=1$. The dominant change in the survival probability for each neutrino is then due 
to the differing scale heights, $h$, (and hence differing jump probabilities) which each neutrino sees. The average over the ensemble of density profiles seen by many neutrinos amounts to an average over $h$.

These calculations find that helioseismic waves have no detectable effect on MSW oscillations, even under optimistic assumptions concerning their amplitudes. This conclusion turns out to rely in an important way on not using a Master-equation-based calculation, because those waves whose amplitudes can be large enough to affect the neutrino resonance, also have correlation lengths (wavelengths) which are much larger than $\ell_{\text {crit }}$. More specifically:

1. Those helioseismic waves ( $p$-waves) which have already been observed at the solar surface generally decrease in amplitude as one moves into the sun, with the result that their amplitude is too small in the MSW resonance region to produce observable effects.

2. The main class of waves ( $g$-waves) which are presently undetected are also too small to affect neutrino resonances if they carry energies which are similar to the $p$-waves which have been seen. This is so even though $g$-waves grow in amplitude with increasing solar depth.

3. A particular class of $g$-waves is believed to be overstable, with runaway evolution once they are sufficiently excited. Although the endpoint of the resulting runaway behaviour remains controversial, it has been argued [14] that the energy in these modes could be as much as $10^{10}$ times larger than what is in other modes. If the generalized Parke equation were used to propagate neutrinos through these waves, one would predict detectable deviations from MSW oscillations. Unfortunately, this prediction is incorrect since the wavelengths of the overstable modes are too large to permit a small- $\ell$ calculation.

\section{Acknowledgements}

The work described here is the result of very stimulating and pleasant collaborations with Denis Michaud and with Peter Bamert, to both of whom I am very grateful. Our principal funding comes from the Natural Sciences and Engineering Council of Canada, with some additional funds being provided by les Fonds pour la Formation de Chercheurs et l'Aide à la Récherche du Québec.

\section{References}

[1] R. Davis, D.S. Harmer and K.C. Hoffman, Phys. Rev. Lett. 20 (1968) 1205; J.K. Rowley et.al., in Solar Neutrinos and Neutrino Astronomy, AIP Conference Proceedings number 126, edited by M.L. Cherry, W.A. Fowler and K. Lande, (1985); K.S. Hirata et.al., Phys. Rev. Lett. 65 (1990) 
1297; P. Anselmann et.al., Phys. Lett. 327B (1994) 377; J.N. Abdurashitov et.al., Phys. Lett. 328B (1994) 234.

[2] The following references provide excellent reviews: J.N. Bahcall, Neutrino Astrophysics, Cambridge University Press, 1989; S. Turck-Chièze et.al., Phys. Rep. 230 (1993) 57; W. Haxton, Ann. Rev. of Astr. and Astrophys. 33 (1995) 459.

[3] A. Cumming and W.C. Haxton, Phys. Rev. Lett. 77 (1996) 4286 nucl-th/9608045; and W. Haxton, this volume.

[4] L. Wolfenstein, Phys. Rev. D17 (1978) 2369; V. Barger, K. Whisnant, S. Pakvasa and R.J.N. Phillips, Phys. Rev. D22 (1980) 2718; P. Langacker, J.P. Leville and J. Sheiman, Phys. Rev. D27 (1983) 1228; S.P. Mikheyev and A. Yu. Smirnov, Sov. Phys. Usp. 29 (1986) 1155; Yad. Fiz. 42 (1985) 1441 [Sov. J. Nucl. Phys. 42 (1985) 913]; Nuovo Cimento 9 (1986) 17; S.P. Rosen and J.M. Gelb, Phys. Rev. D34 (1986) 969; H. Bethe, Phys. Rev. Lett. 56 (1986) 1305; W. Haxton, Phys. Rev. Lett. 57 (1986) 1271; A.J. Baltz and J. Weneser, Phys. Rev. D37 (1988) 3364; P.D. Mannheim, Phys. Rev. D37 (1988) 1935.

[5] R.F. Sawyer, Phys. Rev. D42 (1990) 3908.

[6] F.N. Loreti and A.B. Balantekin, Phys. Rev. D50 (1994) 4762 (nucl-th/9406003); F.N. Loreti, Y.Z. Qian, G.M. Fuller and A.B. Balantekin, Phys. Rev. D52 (1995) 6664 astro-ph/9508106); E. Torrente Lujan, preprint BUTP-96-8 (hep-ph/9602398).

[7] H. Nunokawa, A. Rossi, V.B. Semikoz and J.W.F. Valle, Nucl. Phys. B472 (1996) 495 hepph/9602307); A.B. Balantekin, J.M. Fetter and F.N. Loreti, Phys. Rev. D54 (1996) 3941 (astro$\mathrm{ph} / 9604061$.

[8] C. P. Burgess and D. Michaud, Ann. Phys. (NY) 256 (1997) 1, (hep-ph/9606295); and contribution to the proceedings of the 17th International Conference on Neutrino Physics and Astrophysics (NEUTRINO '96), ed. by K. Enqvist, K. Huitu and J. Maalampi, Helsinki, World Scientific, (hepph/9611368), 1996.

[9] 3) E. Torrente-Lujan, preprint BUTP-96-8, (hep-ph/9602398).

[10] P. Bamert, C. P. Burgess and D. Michaud, preprint (hep-ph/9707542), Phys. Rev. D (to appear).

[11] H.-T. Janka, W. Keil, G.Raffelt and D.Seckel, Phys. Rev. Lett. 76 (1996) 2621.

[12] A very readable review is: Jorgen Christensen-Dalsgaard, These Lecture Notes, are available on the internet at http://bigcat.obs.aau.dk/ jcd/oscilnotes/. 
[13] S.J. Parke, Phys. Rev. Lett. 57 (1986) 1275.

[14] P. Kumar, E.J. Quataert and J.N. Bahcall, Astrophysical Journal 458, (1996), L83.; P. Kumar and J. Goodman, Ap. J. in press, (1996). 